\title{
A Model of Atherosclerosis using Nicotine with Balloon Overdilation in a Porcine
}

\section{Munki Kim}

Cardiovascular convergence Center of Chonnam National University Hospital

\section{Han Byul Kim}

Cardiovascular convergence Center of Chonnam National University Hospital

\section{Dae Sung Park}

Korea Cardiovascular Stent Research Institute

\section{Kyung Hoon Cho}

Chonnam National University Hospital

\section{Dae Young Hyun}

Chonnam National University Hospital

\section{Hae Jin Kee}

Cardiovascular convergence Center of Chonnam National University Hospital

\section{Young Joon Hong}

Chonnam National University Hospital

MyungHo Jeong ( $\nabla$ trikkk01@naver.com )

Chonnam National University Hospital

\section{Research Article}

Keywords: Pigs , cardiovascular , coronary atherosclerosis models

Posted Date: March 1st, 2021

DOI: https://doi.org/10.21203/rs.3.rs-254088/v1

License: (c) (i) This work is licensed under a Creative Commons Attribution 4.0 International License.

Read Full License 


\section{A model of atherosclerosis using nicotine with balloon overdilation in a porcine}

Munki Kim ${ }^{1}$, Han Byul Kim ${ }^{1}$, Dae Sung Park ${ }^{1,2}$, Kyung Hoon Cho ${ }^{3}$, Dae Young Hyun ${ }^{3}$, Hae Jin Kee ${ }^{1}$, Young Joon Hong ${ }^{3}$, Myung Ho Jeong ${ }^{1,2,3, *}$.

1 The Cardiovascular Convergence Research Center of Chonnam National University Hospital Designated by Korea Ministry of Health and Welfare, Gwangju 61469, Republic of Korea

${ }^{2}$ Korea Cardiovascular Stent Research Institute, Jangsung 57248, Republic of Korea

3 Division of Cardiology of Chonnam National University Hospital, Cardiovascular Convergence Research Center Nominated by Korea Ministry of Health and Welfare, Gwangju, Republic of Korea

Address Correspondence to:

Myung Ho Jeong

The Cardiovascular Convergence Research Center of Chonnam National University Hospital Designated by Korea Ministry of Health and Welfare

Gwangju 61469, Republic of Korea

Tel : +82-62-220-6243

myungho@chollian.net 


\begin{abstract}
Pigs are one of the important experimental animals for cardiovascular research. Few porcine coronary atherosclerosis models have been developed; however, the induction of which requires longer than 6 months. We developed a porcine coronary artery atherosclerosis model using nicotine injection with a balloon overdilation. A coronary balloon was placed in the porcine coronary artery and was over dilated to induce a mechanical injury. Nicotine was administrated via intramuscular injection every day and changes in the coronary artery were observed after 4 weeks. Coronary angiography revealed nicotine injection with a balloon overdilation groups showed narrowing of the coronary artery at the injury site. Combination of balloon and nicotine significantly increased the intimal hyperplasia on optical coherence tomography analysis. Proliferated tunica media was noted in the nicotine injection with balloon overdilation groups. Quantitative analysis showed increased smooth muscle actin alpha (SMA), cluster of differentiation 68 (CD68), and Krüppel-like factor 4 (KLF4) in the nicotine injection with balloon overdilation groups. The immunohistochemistry results showed CD68-positive cells displayed SMA- and KLF4-positive reactivity in the border zone of intimal hyperplasia. Our results show that nicotine injection with balloon overdilation can induce an atherosclerosis lesion within one month, which mimics the human atherosclerosis.
\end{abstract}




\section{Introduction}

Atherosclerosis is defined as a chronic progressive inflammatory disease. Its main components include the accumulation of proliferative alpha smooth muscle actin (a-SMA), large numbers of macrophage, a necrotic core containing rich lipids, and cellular debris [1,2]. The precise reasons for the rupture or erosion of an unstable atherosclerosis remain unknown; arthrosclerosis lesions can rupture and induce thrombotic events that lead to myocardial infarction or stroke, the major cause of human death in worldwide [3].

Nicotine is the main ingredient of cigarette and smoking is the single most important risk factor for tumorigenesis, cardiovascular disease, and peripheral vascular diseases [4,5]. Nicotine changed proliferation of the endothelial cells and enhancement of angiogenesis $[6,7,8]$. It also activates atherosclerosis associated factors in endothelial cells, such as, endothelial nitric oxide synthase, tissue-type plasminogen activator, platelet-derived growth factor, and basic fibroblast growth factor In the presence of vascular disease $[9,10]$. It also changes endothelial cell morphology, induces inflammatory responses within endothelial cells and increases endothelial cell death $[8,11]$.

Pigs have been studied as models of human cardiovascular diseases and have been used to validate cardiovascular medical devices. Their cardiovascular systems, artery distribution, and heart size are similar to those of humans [12]. They spontaneously develop coronary artery atherosclerosis because of their lipid profiles $[13,14]$. Despite these advantages, use of pig as an experimental animal model is restricted. Complications related to induction of atherosclerosis also create restrictions. Further, a series of studies demonstrated a variety of atherosclerosis porcine models, but the induction of atherosclerotic lesions requires longer than 6 months $[15,16]$.

This study aimed to develop a rapid atherosclerosis porcine model using nicotine injection 
and balloon overdilation. 


\section{Results}

\section{Optical coherence tomography analysis}

Optical coherence tomography (OCT) analysis was performed to identify the changes that occurred in the intima of the coronary artery. The control group showed thin and compact coronary artery wells (Fig. 1A). All groups of treatment showed increase of the percent intimal hyperplasia in the OCT images (Fig. 1B-D). In the volumetric analysis of OCT, percent intimal hyperplasia significantly increased in the nicotine injection with balloon overdilation groups $(26.38 \pm 3.81 \%$ in control group vs. $36.15 \pm 7.65 \%$ in balloon overdilation group vs. $42.07 \pm 6.75 \%$ in nicotine injection group vs. $60.86 \pm 7.20 \%$ in nicotine with balloon overdilation group) (Fig. 1E).

\section{Histopathologic findings after nicotine injection with balloon over-dilation}

Each dose of nicotine was administrated to the pigs whose coronary arteries were ruptured by the coronary balloon inflation to achieve intimal hyperplasia of the coronary arteries. Unlike simple nicotine injection or balloon overdilation, $0.05 \mathrm{mg} / \mathrm{kg}$ nicotine injection with balloon overdilation induced intimal hyperplasia (Fig. 2A, D). The $0.25 \mathrm{mg} / \mathrm{kg}$ nicotine injection with balloon overdilation showed significant intimal hyperplasia of the tunica media covering the internal elastic membrane (Fig. 2B, E). The higher dose $(0.5 \mathrm{mg} / \mathrm{kg})$ nicotine injection with balloon overdilation group exhibited similar intimal hyperplasia as that of the $0.25 \mathrm{mg} / \mathrm{kg}$ nicotine groups (Fig. 2C, F).

\section{The expression of KLF4 and CD68 expression in the coronary arteries}

Western blot analysis of the coronary artery samples was performed to quantify changes in KLF4 and CD68 expression (Fig. 3A). Samples from the control group did not express CD68, whereas those from the nicotine injection with balloon overdilation groups showed 
significantly increased CD68 expression (Fig. 3B). Similar KLF4 expression was observed in the control and balloon overdilation groups, and nicotine injection increased the KLF4 expression. However, the nicotine injection with balloon overdilation group had significantly increased KLF4 expression than the other groups (Fig. 3C).

\section{Intimal hyperplasia via KLF4 after nicotine injection with balloon overdilation}

In the nicotine injection with balloon overdilation groups, immunohistochemical analysis was performed to identify the expression of SMA, CD68, and KLF4. In hyperplastic lesions, SMA positive reactive cells and CD68 positive cells were separately observed (Fig. 4A-D). An examination at a higher magnification board zone revealed SMA and CD68 co-positive cells (Fig. 4E-H). Moreover, the CD68 positive cells co-expressed KLF4 (Fig. 4I-L). 


\section{Discussion}

According to our experiments, the nicotine injection with balloon overdilation is a novel approach to induce porcine coronary atherosclerosis. This animal model can serve as a suitable pathological model for future human atherosclerosis research.

Several small and large animal models of atherosclerosis have been developed in research field. Although small animal models, such as those of mice, rats, and rabbits, have provided valuable information about cardiovascular diseases, there are limitations to the clinical application of these animal models. For example, mice atherosclerotic models are relatively low cost and feature easy breeding and genetic manipulation compared to the large animal models. However, these models naturally develop atherosclerotic lesions not in the coronary arteries, but in the aortic root, and they do not develop thick fibrous cap atherosclerotic lesions $[1,18]$. Small size of the model is another key limitation. Their size restricts the use of the model in the development of human coronary devices (such as coronary stent) and the use of imaging techniques or tools. Pigs have been used to develop large animal atherosclerosis models. However, previous porcine coronary models had limitation, they required extended time for lesion induction, featured lesions of varying severities, and had unpredictable plaque locations[12,19,20].

Balloon overdilation induced luminal stenosis in the porcine coronary artery. Percutaneous coronary intervention was performed to open blocked or stented coronary arteries. During this process, mechanical stimulation to the artery is inevitable, and the experimental study was conducted to identify the response of the injured coronary artery to the balloon overdilation. Balloon overdilation mechanically damaged the coronary artery and induced endothelial denudation. Injured arteries exposed the blood and induced thrombus formation and inflammatory response. Consequently, injured arteries formed stenotic lesion [21,22]. 
Our results are consistent with those of the previous studies. Balloon injured porcine coronary arteries demonstrated dissection and luminal stenosis.

Nicotine injection can induce the proliferation of endothelial cells within the coronary arteries. The coronary arteries consist of the tunica intima, media, and adventitia among these, the tunica intima is covered with the vascular smooth muscle cells (VSMCs), which express the transmembrane ligand-gated ion channels nicotinic acetylcholine receptors $[13,23]$. Nicotine is the major hazardous component of cigarettes, and a series of studies has demonstrated the atherogenic effect of nicotine. Nicotine exposure disturbs the normal expression of endothelial cell derived platelet-derived growth factor, basal fibroblastic growth factor, and vascular endothelial growth factor $[9,24,25]$. It also promotes the migration and proliferation of the VSMCs in the coronary arteries and changes the VSMC phenotype from contractile to synthetic [25]. Through these mechanisms, nicotine accelerates the formation of atherosclerotic characteristics of the intima lesion. Our results showed that the proliferation of the tunica intima is dependent on nicotine dose.

Nicotine injection with balloon overdilation induces atherosclerotic lesion by activating KLF4 expression. Atherosclerosis is a chronic inflammatory disease with narrowing of the coronary arteries. The coronary angiogram and OCT results in our study showed the significant stenosis of the porcine coronary arteries in the nicotine injection with balloon overdilation groups. The narrowing was closely related to characteristic plaque lesions. Pathological studies have reported a large numbers of macrophages marker positive cells, such as CD68, in the plaque resulting from the VSMCs, which were normally expressed in the coronary arteries [3,26]. Phenotypic switching, specifically from VSMCs to macrophages, has been considered an important mechanism for the development of atherosclerosis, and recent studies have described the role of KLF4 in the phenotypic switching [26,27]. KLF4 
regulates various cell functions, such as proliferation, differentiation, apoptosis, embryonic development, and tissue homeostasis [28]. In the VSMCs, KLF4 expression is associated with VSMC phenotypic switching. KLF4 down regulation delayed phenotypic switching of the VSMCs under stressful in vitro conditions [29,30]. KLF4 knockout mice demonstrated decreased differentiation of the VSMCs to macrophage-like cells and increased plaque stability and fibrous cap thickness compared to the wild type mice [31]. In our study results, balloon overdilation or nicotine injection slightly increased the expression of KLF4 and CD68 in the porcine coronary arteries. Nicotine injection with balloon overdilation significantly increased the expression of KLF4 and CD68. The immunohistochemistry results demonstrated that, CD68 positive cells co-expressed SMA and KLF4 in the intimal hyperplasia lesions. These results demonstrated that nicotine injection with balloon overdilation can induce atherosclerosis lesions in the porcine coronary arteries, creating a model that closely mimics the human atherosclerosis disease mechanism. The nicotine injection with balloon overdilation porcine model is a novel atherosclerosis model that accelerates the cardiovascular devices development. 


\section{Methods}

\section{Animal and study groups}

The Institutional Animal Care and Use Committee (IACUC) of Chonnam National University Hospital (IACUC approval No.CNUHIACUC-18006) approved this animal study, and it conforms to the Guide for the Care and Use of Laboratory Animals published by the US National Institutes of Health (NIH Publication No. 85-23, revised 1996) and the ARRIVE (Animal Research: Reporting of In Vivo Experiments) guidelines for reporting animal research [17]. Study animals were Yorkshire X Landrace F1 crossbred castrated male swine $(15-20 \mathrm{~kg})$ provided by Chuwol grandparent farm located in the southwest of the Republic of Korea. Study animals were randomly allocated to group 1 [negative control, $n=10$ ], group 2 [balloon overdilation, $\mathrm{n}=10]$, group $3[0.05 \mathrm{mg} / \mathrm{kg}$ nicotine injection, $\mathrm{n}=10]$, group 4 [0.25 $\mathrm{mg} / \mathrm{kg}$ nicotine injection, $\mathrm{n}=10]$, group 5 [0.5 mg/kg nicotine injection, $\mathrm{n}=10]$, group 6 [0.05 $\mathrm{mg} / \mathrm{kg}$ nicotine injection with balloon overdilation, $\mathrm{n}=10]$, group $7[0.25 \mathrm{mg} / \mathrm{kg}$ nicotine injection with balloon overdilation, $\mathrm{n}=10]$, and group $8[0.5 \mathrm{mg} / \mathrm{kg}$ nicotine injection with balloon overdilation, $\mathrm{n}=10]$.

\section{Nicotine injection and balloon overdilation procedures}

Aspirin $100 \mathrm{mg}$ and clopidogrel $75 \mathrm{mg}$ per day were administered to the study animals for 5 days before the procedure. On the day of procedure, pigs were anesthetized with zolazepam and tiletamine $\left(2.5 \mathrm{mg} / \mathrm{kg}\right.$, Zoletil50 ${ }^{\circledR}$, Virvac, Caros, France), xylazine (3 mg/kg, Rompun ${ }^{\circledR}$,

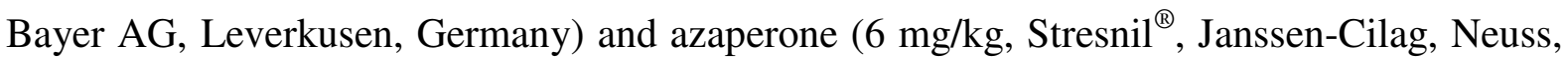
Germany). Continuous oxygen was supplied through an oxygen mask. After subcutaneous $2 \%$ lidocaine injection, the left carotid artery was surgically exposed, and a 7-French (Fr) sheath was inserted. Continuous hemodynamic and surface electrocardiographic monitoring was 
maintained throughout the procedure. Subsequently 5,000 units of heparin was administered intravenously as a bolus and the target coronary artery was engaged using standard 7-Fr guide catheters and baseline angiograms were performed using nonionic contrast agent in two orthogonal views. Balloon overdilation was performed by inflating the balloon with the resulting balloon-to-artery diameter ratio in the range of $1.3-1.4: 1$ to achieve maximum luminal patency. Coronary angiograms were obtained immediately after balloon overdilation. After the balloon overdilation procedures, the pigs were administered $0.05 \mathrm{mg} / \mathrm{kg}, 0.25$ $\mathrm{mg} / \mathrm{kg}$, and $0.5 \mathrm{mg} / \mathrm{kg}$ nicotine via intramuscular routes daily for one month. One month later, the pigs underwent repeat angiography in the same orthogonal views and were euthanized with an intracoronary injection of potassium chloride $(15 \%, 20 \mathrm{~mL})$. The samples of the coronary arteries were pressure-perfusion fixed at $70 \mathrm{mmHg}$ in $10 \%$ neutral buffered formalin for 24-48 hours.

\section{Optical coherence tomography analysis}

Optical coherence tomography (OCT) was performed using a 2.7 Fr C7 Dragonfly ${ }^{\mathrm{TM}}$ imaging catheter (LightLab Imaging Inc., Westford, MA, USA). The catheter was placed in the distal native artery to the balloon overdilated lesion, and automatic pullback at a speed of $20 \mathrm{~mm} / \mathrm{s}$ was done during continuous automatic flushing of iodixanol (Visipaque ${ }^{\mathrm{TM}} 320 \mathrm{mg} \mathrm{I} / \mathrm{mL}$, GE Healthcare, Amersham, UK) at the rate of $2-5 \mathrm{~mL} / \mathrm{s}$ using a Medrad injector (Medrad Inc., Warrendale, PA, USA). Qualitative and quantitative measurements were achieved using OCT to assess the balloon overdilation area at one-month follow-up. OCT images were analyzed at a $1 \mathrm{~mm}$ distance in a blinded fashion. Volumetric analysis was performed to measure the lumen area and intimal hyperplasia area. 


\section{Histopathologic and immunohistochemical analyses}

The fixed coronary artery samples were placed perpendicular to the direction of blood flow at $5 \mathrm{~mm}$ intervals and were embedded in paraffin. The coronary artery samples were sectioned at a thickness of $5 \mu \mathrm{m}$ on a rotary microtome for histopathologic and immunohistochemical analyses. Hematoxylin and eosin staining was performed for the histopathologic analysis. Immunohistochemical analysis was carried out to identify the proteins expression. Nonspecific reactivity was blocked with $3 \%$ fetal bovine serum in phosphate-buffered saline for 60 minutes. Immunohistochemistry was performed using anti-rabbit monoclonal alpha smooth muscle actin (SMA, 1:100; Abcam, Milton, Cambridge, UK), anti-rabbit polyclonal Krüppel-like factor 4(KLF4, 1:100; LSBio, Seattle, Washington, USA), and anti-mouse monoclonal cluster of differentiation 68 (CD68, 1:20; Invitrogen, St. Louis, MO, USA) antibodies. The secondary antibodies were streptavidin Alexa Fluor ${ }^{\circledR}$ 594-conjugated antimouse immunoglobulin $\mathrm{G}(\mathrm{IgG})\left(1: 400\right.$; Invitrogen) and streptavidin Alexa Fluor ${ }^{\circledR}$ 488conjugated anti-rabbit IgG (1:400; Invitrogen) for fluorescence microscopy. Images were captured using Nikon eclipse 80i fluorescence microscopy.

\section{Western blot analysis}

The porcine coronary arteries (negative control, balloon overdilation, $0.25 \mathrm{mg} / \mathrm{kg}$ nicotine injection, and $0.25 \mathrm{mg} / \mathrm{kg}$ nicotine injection with balloon overdilation) were collected to evaluate changes in protein expression. Samples were washed with cold phosphate-buffered saline and total cell lysates were prepared using lysis buffer (1 $\mathrm{M}$ Tris- $\mathrm{HCl}$ [pH 8.0], $5 \mathrm{M}$ $\mathrm{NaCl}, 1 \% \mathrm{NaN}_{3}, 10 \%$ sodium dodecyl sulfate, $10 \% \mathrm{NP}-40$, and $0.5 \% \mathrm{C}_{24} \mathrm{H}_{39} \mathrm{NaO}_{4}$ ). Equal sample amounts were loaded onto a $10 \%$ polyacrylamide gel. Following electrophoresis, proteins were transferred to polyvinylidene membranes. Non-specific binding was blocked with $5 \%$ skim milk in $0.1 \%$ Tween 20 for 30 minutes. The membranes were incubated with 
anti-rabbit polyclonal KLF4 (1:1,000; LSBio), anti-mouse monoclonal CD68 (1:500; Invitrogen), and anti-rabbit polyclonal glyceraldehyde 3-phosphate dehydrogenase (GAPDH, 1:1,000; Santa Cruz, Dallas, Taxas, USA) antibodies overnight at $4^{\circ} \mathrm{C}$. After washing five times, the membranes were incubated with horseradish peroxidase-conjugated secondary antibodies, and proteins were detected using an enhanced chemiluminescence reagent (Anigen, Seoul, Korea). Densitometry was performed using the Image $\mathbf{J}$ software, and statistical analysis was carried out using Instat ${ }^{\circledR}$ software (Graphpad, San Diego, CA, USA).

\section{Statistical analysis}

Data are represented as means \pm standard deviations of at least three independent experiments. Statistical differences were tested using analyses of variance, which were performed using GraphPad Prism 5.0 (Graphpad). $P$ values less than 0.05 were considered statistically significant. 


\section{References}

1. Tabas, I., Williams, K. J. \& Boren, J. Subendothelial lipoprotein retention as the initiating process in atherosclerosis: update and therapeutic implications. Circulation 116, 1832-1844 (2007).

2. Virmani, R., Kolodgie, F. D., Burke, A. P., Farb, A. \& Schwartz, S. M. Lessons from sudden coronary death: a comprehensive morphological classification scheme for atherosclerotic lesions. Arterioscler. Thromb. Vasc. Biol. 20, 1262-1275 (2000).

3. Libby, P., Ridker, P. M. \& Hansson, G. K. Progress and challenges in translating the biology of atherosclerosis. Nature 473, 317-325 (2011).

4. Lakier, J. B. Smoking and cardiovascular disease. Am. J. Med. 93, 8S-12S (1992).

5. Powell, J. T. Vascular damage from smoking: disease mechanisms at the arterial wall. Vasc. Med. 3, 21-28 (1998).

6. Calderon, L. E. et al. Bromoenol Lactone Attenuates Nicotine-Induced Breast Cancer Cell Proliferation and Migration. PLoS One 10, e0143277 (2015).

7. Heusch, W. L. \& Maneckjee, R. Signalling pathways involved in nicotine regulation of apoptosis of human lung cancer cells. Carcinogenesis 19, 551-556 (1998).

8. Wang, C. N. et al. Role of perivascular adipose tissue in nicotineinduced endothelial cell inflammatory responses. Mol. Med. Rep. 14, 5713-5718 (2016).

9. Conklin, B. S. et al. Effects of nicotine and cotinine on porcine arterial endothelial cell function. J. Surg. Res. 95, 23-31 (2001). 
10. Cucina, A. et al. Nicotine reorganizes cytoskeleton of vascular endothelial cell through platelet-derived growth factor BB. J. Surg. Res. 92, 233-238 (2000).

11. Lin, S. J., Hong, C. Y., Chang, M. S., Chiang, B. N. \& Chien, S. Long-term nicotine exposure increases aortic endothelial cell death and enhances transendothelial macromolecular transport in rats. Arterioscler. Thromb. 12, 1305-1312 (1992).

12. Gerrity, R. G., Natarajan, R., Nadler, J. L. \& Kimsey, T. Diabetes-induced accelerated atherosclerosis in swine. Diabetes 50, 1654-1665 (2001).

13. Li, S. et al. Nicotinic acetylcholine receptor alpha7 subunit mediates migration of vascular smooth muscle cells toward nicotine. J. Pharmacol. Sci. 94, 334-338 (2004).

14. Skold, B. H., Getty, R. \& Ramsey, F. K. Spontaneous atherosclerosis in the arterial system of aging swine. Am. J. Vet. Res. 27, 257-273 (1966).

15. Hamamdzic, D. \& Wilensky, R. L. Porcine models of accelerated coronary atherosclerosis: role of diabetes mellitus and hypercholesterolemia. J. Diabetes Res. 2013, 761415 (2013).

16. Hedayat, A. F. et al. Peripheral vascular atherosclerosis in a novel PCSK9 gain-offunction mutant Ossabaw miniature pig model. Transl. Res. 192, 30-45 (2018).

17. Kilkenny, C., Browne, W. J., Cuthill, I. C., Emerson, M. \& Altman, D. G. Improving bioscience research reporting: the ARRIVE guidelines for reporting animal research. PLoS Biol. 8, e1000412 (2010).

18. Yang, X. et al. Identification and validation of genes affecting aortic lesions in mice. $J$. Clin. Invest. 120, 2414-2422 (2010). 
19. Al-Mashhadi, R. H. et al. Familial hypercholesterolemia and atherosclerosis in cloned minipigs created by DNA transposition of a human PCSK9 gain-of-function mutant. Sci. Transl. Med. 5, 166ra1 (2013).

20. Prescott, M. F., McBride, C. H., Hasler-Rapacz, J., Von Linden, J. \& Rapacz, J. Development of complex atherosclerotic lesions in pigs with inherited hyper-LDL cholesterolemia bearing mutant alleles for apolipoprotein B. Am. J. Pathol. 139, 139-147 (1991).

21. Steele, P. M. et al. Balloon angioplasty. Natural history of the pathophysiological response to injury in a pig model. Circ. Res. 57, 105-112 (1985).

22. Wilensky, R. L. et al. Vascular injury, repair, and restenosis after percutaneous transluminal angioplasty in the atherosclerotic rabbit. Circulation 92, 2995-3005 (1995).

23. Zhou, J., Li, Y. S. \& Chien, S. Shear stress-initiated signaling and its regulation of endothelial function. Arterioscler. Thromb. Vasc. Biol. 34, 2191-2198 (2014).

24. Conklin, B. S., Zhao, W., Zhong, D. S. \& Chen, C. Nicotine and cotinine up-regulate vascular endothelial growth factor expression in endothelial cells. Am. J. Pathol. 160, 413418 (2002).

25. Yoshiyama, S. et al. Nicotine exposure alters human vascular smooth muscle cell phenotype from a contractile to a synthetic type. Atherosclerosis $\mathbf{2 3 7}$, 464-470 (2014).

26. Owens, G. K., Kumar, M. S. \& Wamhoff, B. R. Molecular regulation of vascular smooth muscle cell differentiation in development and disease. Physiol. Rev. 84, 767-801 (2004). 
27. Deaton, R. A., Gan, Q. \& Owens, G. K. Sp1-dependent activation of KLF4 is required for PDGF-BB-induced phenotypic modulation of smooth muscle. Am. J. Physiol. Heart Circ. Physiol. 296, H1027-37 (2009).

28. Ghaleb, A. M. \& Yang, V. W. Kruppel-like factor 4 (KLF4): What we currently know. Gene 611, 27-37 (2017).

29. Pidkovka, N. A. et al. Oxidized phospholipids induce phenotypic switching of vascular smooth muscle cells in vivo and in vitro. Circ. Res. 101, 792-801 (2007).

30. Yoshida, T., Gan, Q. \& Owens, G. K. Kruppel-like factor 4, Elk-1, and histone deacetylases cooperatively suppress smooth muscle cell differentiation markers in response to oxidized phospholipids. Am. J. Physiol. Cell. Physiol. 295, C1175-82 (2008).

31. Shankman, L. S. et al. KLF4-dependent phenotypic modulation of smooth muscle cells has a key role in atherosclerotic plaque pathogenesis. Nat. Med. 21, 628-637 (2015). 


\section{Acknowledgements}

This research was supported by the National Research Foundation of Korea (NRF2019R1F1A1049158).

Author information

\section{Affiliations}

The Cardiovascular Convergence Research Center of Chonnam National University Hospital Designated by Korea Ministry of Health and Welfare, Gwangju 61469, Republic of Korea

Munki Kim, Han Byul Kim, Myung Ho Jeong

Korea Cardiovascular Stent Research Institute, Jangsung 57248, Republic of Korea

Dae Sung Park, Myung Ho Jeong

Division of Cardiology of Chonnam National University Hospital, Cardiovascular Convergence Research Center Nominated by Korea Ministry of Health and Welfare, Gwangju, Republic of Korea

Kyung Hoon Cho, Dae Young Hyun, Hae Jin Kee ${ }^{1}$, Young Joon Hong, Myung Ho Jeong

\section{Contributions}

M.K. and H. K. conceived the idea. M.K., DS. P., KH. C., DY. H. and HJ.K performed the animal experiments and analyzed the data. M.K., YJ. H. and MH. J. wrote the paper. All 
authors reviewed the manuscript.

\section{Corresponding author}

\section{Correspondence to Myung Ho Jeong}

The Cardiovascular Convergence Research Center of Chonnam National University Hospital Designated by Korea Ministry of Health and Welfare

Gwangju 61469, Republic of Korea

Tel : +82-62-220-6243

myungho@chollian.net

\section{Ethic Declarations}

\section{Conflict of interest statement}

The authors declare that they have no known competing financial interests or personal relationships that could have appeared to influence the work reported in this paper 


\section{Figure Legends}

Figure 1. Intimal hyperplasia after balloon overdilation and nicotine injection in the coronary arteries. Optical coherence tomography (OCT) was performed to identify changes in the coronary arteries. (A) The control group showed thin and compact coronary artery wells. (B) The balloon overdilation induced intimal hyperplasia. (C) The $0.25 \mathrm{mg} / \mathrm{kg}$ nicotine injection induced coronary artery intimal hyperplasia. (D) The nicotine injection with balloon overdilation significantly increased the intimal hyperplasia compared to simple balloon or nicotine injection groups. (E) Table shows the results of the volumetric analysis of OCT. Nicotine injection with balloon overdilation groups increased the percentage of intimal area compared to the control groups. The nicotine injection with balloon overdilation groups showed significantly increased percent intimal area compared to the other groups $(26.38 \pm$ 3.81 in control group vs. $36.15 \pm 7.65$ in balloon overdilation group vs. $42.07 \pm 6.75$ in nicotine injection group vs. $60.86 \pm 7.20$ in nicotine with balloon overdilation group). (* $P<0.05$ compared to control groups. \# $P<0.05$ compared to balloon or nicotine injection groups). 
Figure 2. Hyperplasia of the coronary arteries after nicotine injection with balloon overdilation. (A, D) The dose of $0.05 \mathrm{mg} / \mathrm{kg}$ nicotine with balloon overdilation delayed the healing of the injured internal elastic lamina and induced proliferation of the tunica media. (B, E) The dose of $0.25 \mathrm{mg} / \mathrm{kg}$ nicotine injection with balloon overdilation induced clear intimal hyperplasia compared to that seen in the lower dose and simple balloon overdilation. (C, F) The dose of $0.5 \mathrm{mg} / \mathrm{kg}$ nicotine injection with balloon overdilation induced intimal hyperplasia similar to the $0.25 \mathrm{mg} / \mathrm{kg}$ nicotine injection with balloon overdilation. Bar: 100 $\mu \mathrm{m}$ 
Figure 3. Increased expression of Krüppel-like factor 4 (KLF4) and cluster of differentiation 68 (CD68) in the nicotine injection with balloon overdilation groups. (A) Western blot analysis was performed to identify the changes in KLF4 and CD68 expression. (B) In the control groups, CD68 was not expressed in the coronary arteries. Balloon overdilation or nicotine injection increased CD68 expression. The nicotine injection with balloon overdilation groups had significantly increased CD68 expression compared to the other groups. (C) KLF4 expression was similar in the control and balloon overdilation groups and nicotine injection increased KLF4 expression. Nicotine injection with balloon overdilation significantly increased KLF4 expression in the coronary arteries. ${ }^{*} P<0.05$. 
Figure 4. Alpha smooth muscle actin (SMA) positive cells and cluster of differentiation 68 (CD68) in the intimal hyperplasia lesion of nicotine injection with balloon overdilation group. (A-D) In the hyperplastic lesion of nicotine injection with balloon overdilation groups, SMA positive cells and CD68 positive cells were separately observed. (E-H) An examination at higher magnification, board zone revealed SMA and CD68 co positive cells. (I-L) CD68 positive cells co expressed KLF4. 

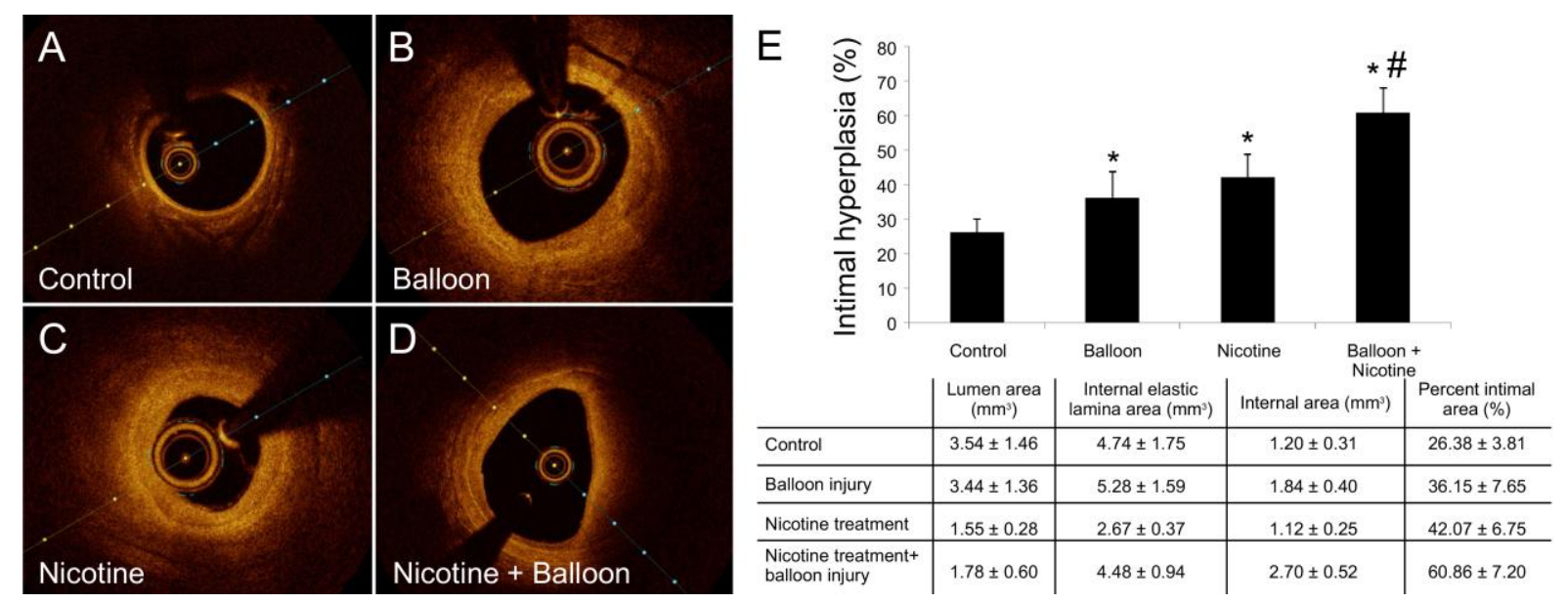

Figure 1. 


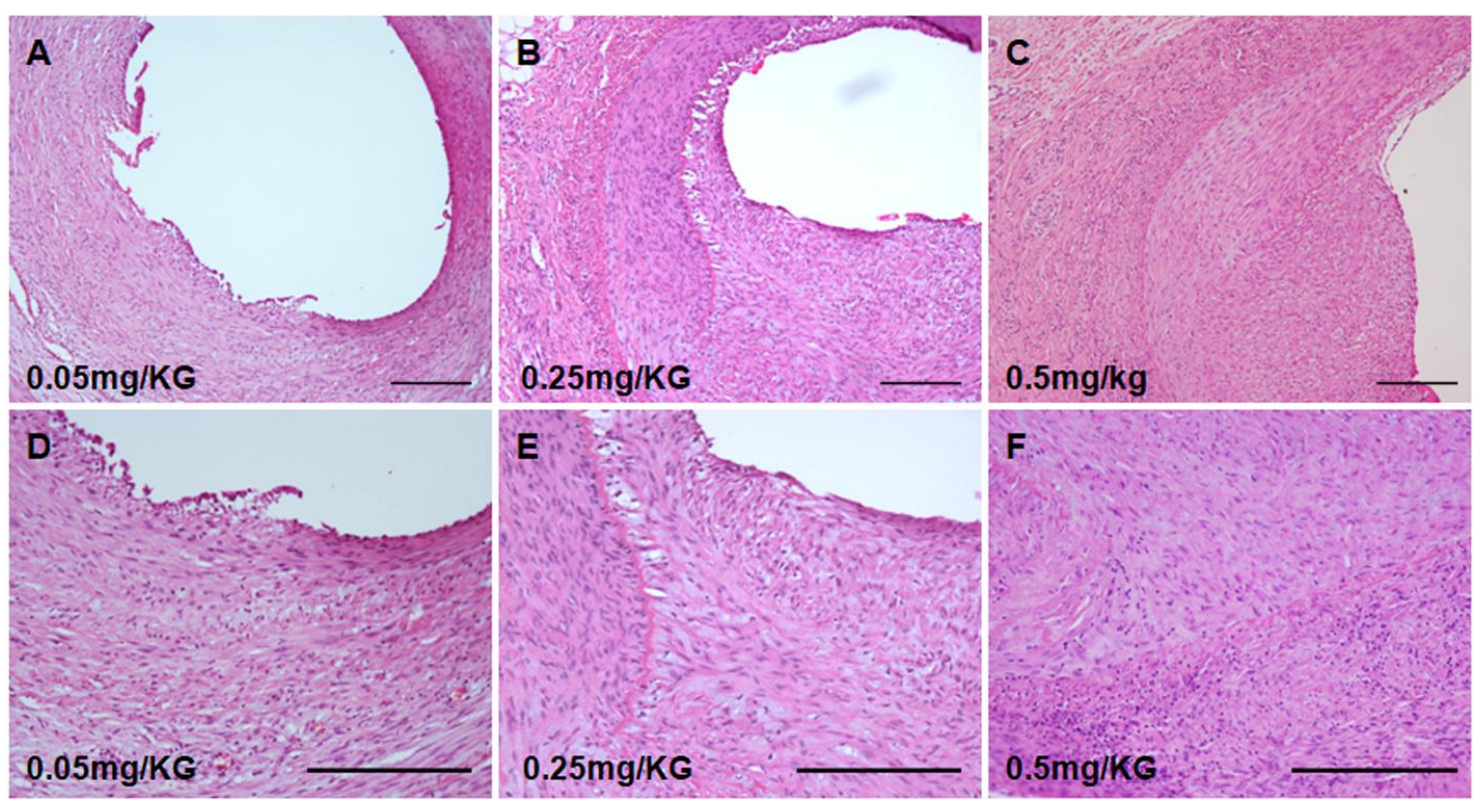

Figure 2. 


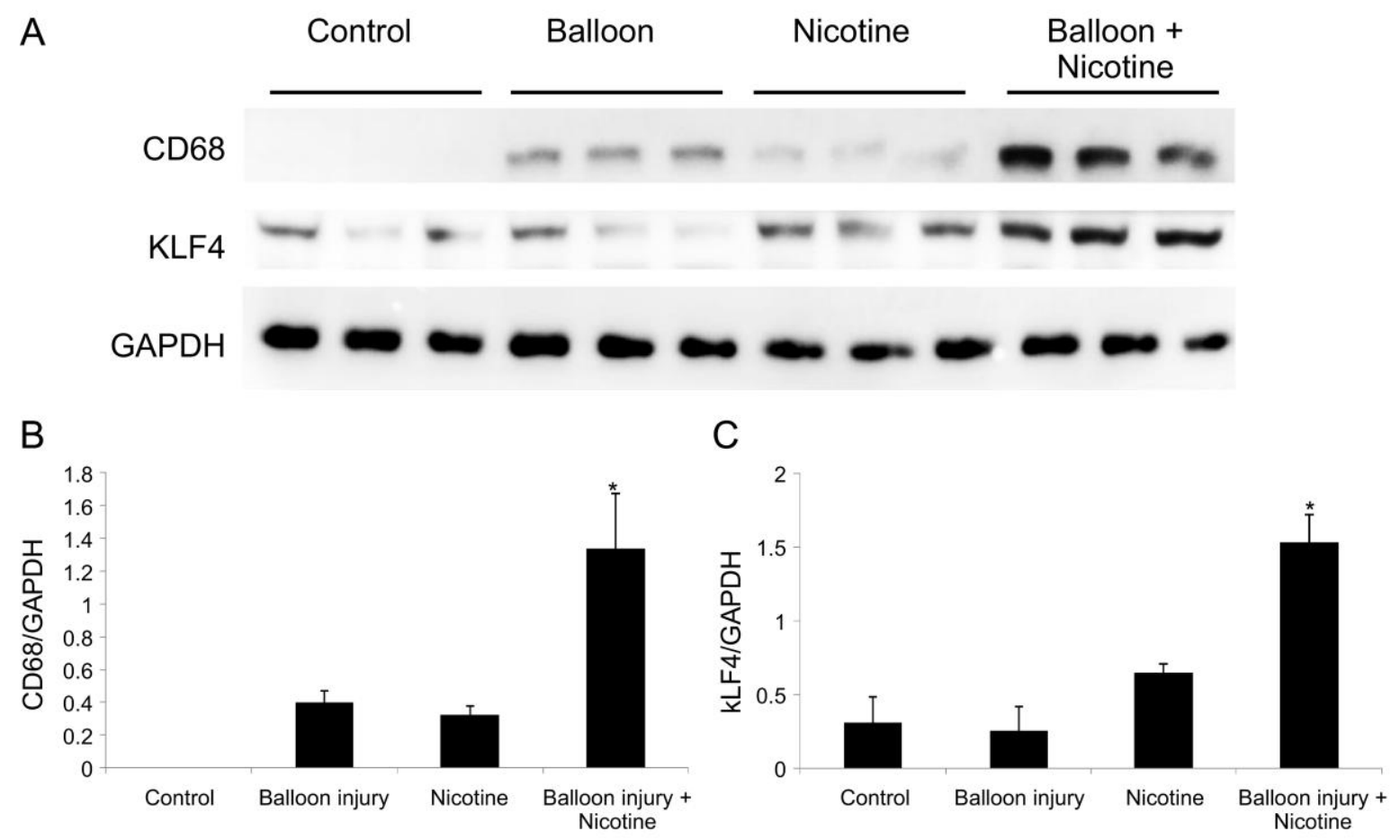

Figure 3. 


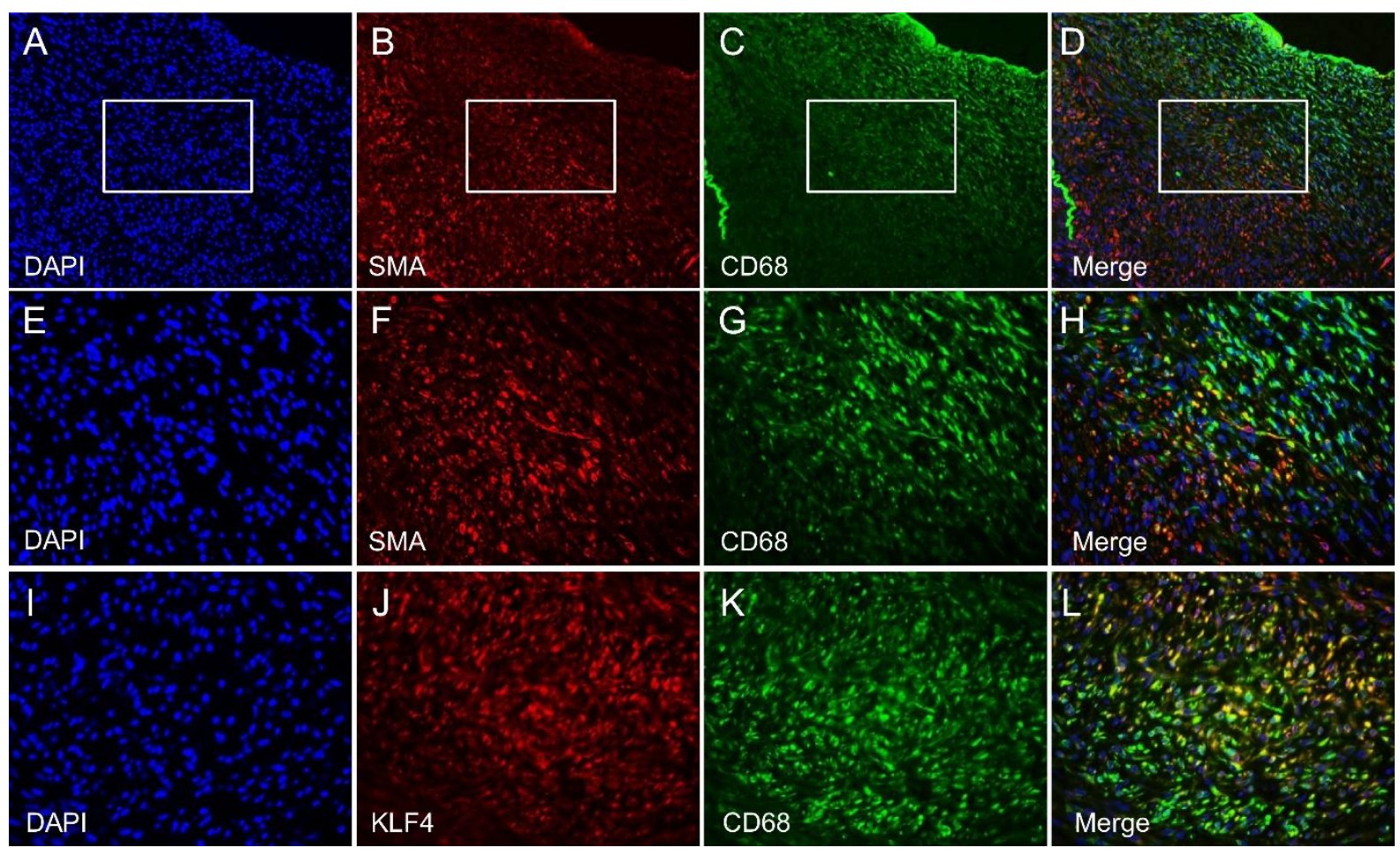

Figure 4. 


\section{Supplementary Information}

\section{Coronary angiographic findings}

Coronary angiography, the gold standard for the assessing coronary artery diseases, was performed to identify the changes occurring in the porcine coronary arteries at 4 weeks after the experiment. The left anterior descending artery and left circumflex artery (LCX) were observed on the baseline coronary angiography (Supplementary Fig.1A, B). A coronary balloon was placed in the LCX and inflated to induce a mechanical injury to the vessel walls (Supplementary Fig.1C, D). Four weeks after the experiment, the simple balloon overdilation group showed no angiographic lesion (Supplementary Fig. 1E). However, the $0.25 \mathrm{mg} / \mathrm{kg}$ nicotine injection with balloon overdilation group exhibited significant narrowing of the coronary arteries in the injured LCX (Supplementary Fig. 1F).

\section{Supplementary data figure legends}

Supplementary Figure 1. Coronary angiographic findings. (A) Base line image shows the distribution of the left anterior descending and left circumflex (LCX) arteries. (B) The balloon overdilation was induced using over inflation of a coronary balloon. (C) One month after the injury, angiography showed the LCX diameter was similar to that at base line. (D-E) After the balloon overdilation, nicotine was administered via intramuscular injection. (F) One month later, angiography showed a clearly narrowed LCX. 


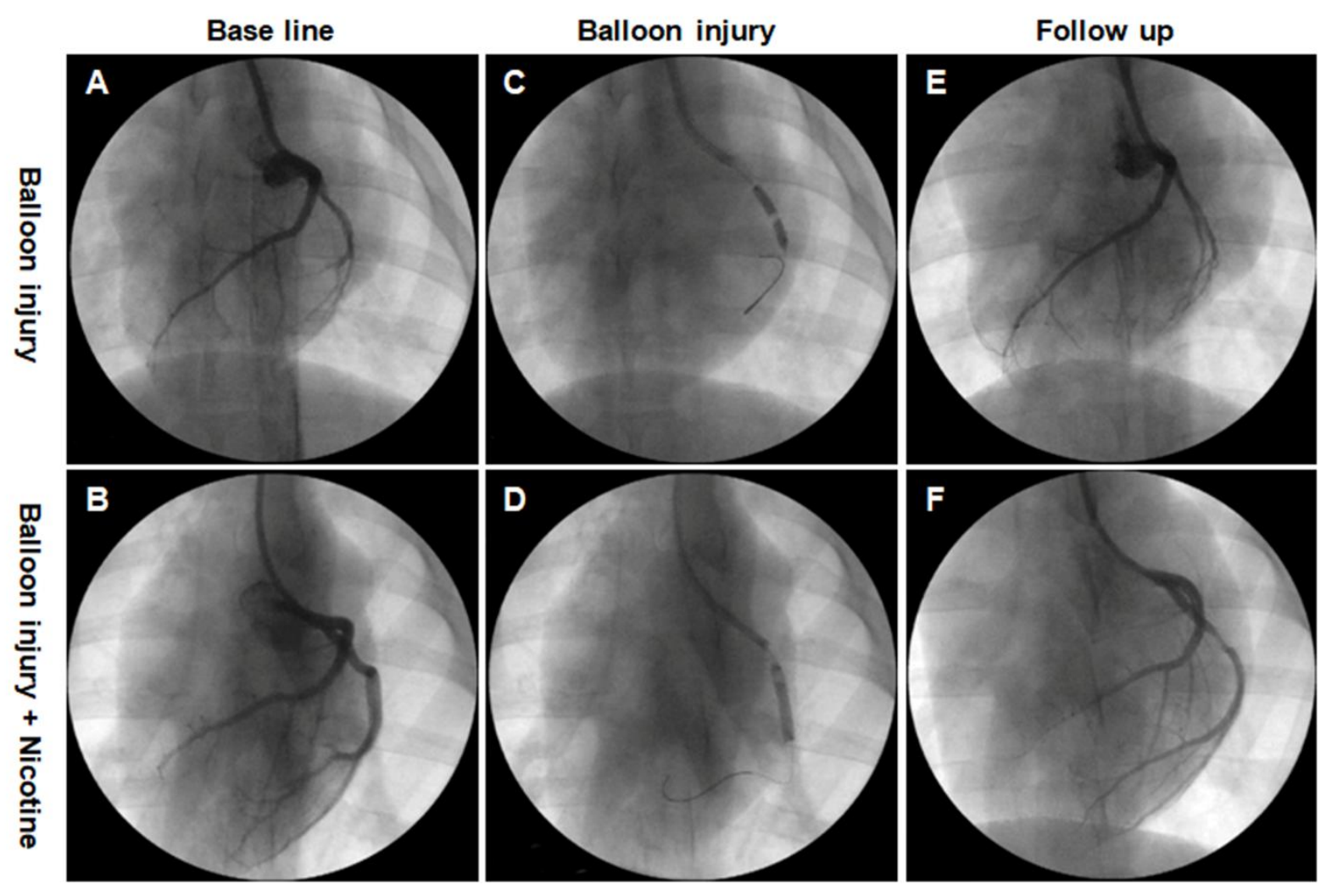

Supplementary Figure 1. 

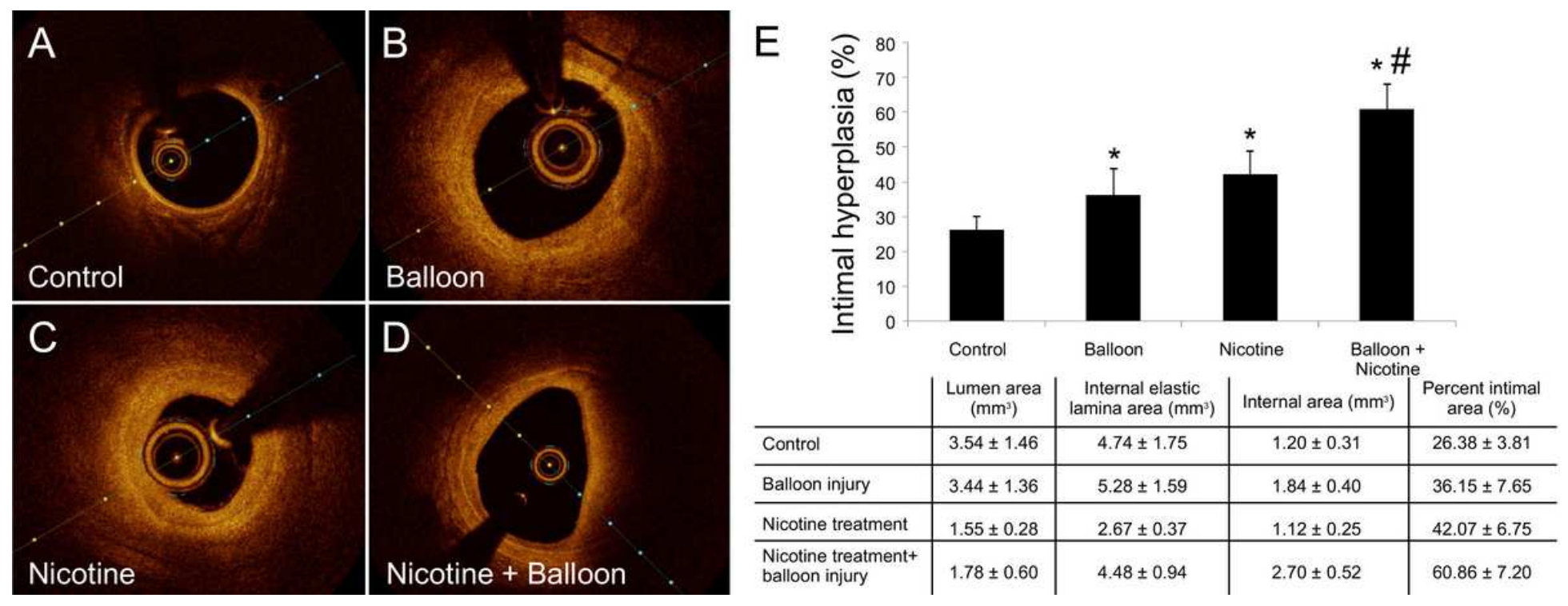

Figure 1

Intimal hyperplasia after balloon overdilation and nicotine injection in the coronary arteries. Optical coherence tomography (OCT) was performed to identify changes in the coronary arteries. (A) The control group showed thin and compact coronary artery wells. (B) The balloon overdilation induced intimal hyperplasia. (C) The $0.25 \mathrm{mg} / \mathrm{kg}$ nicotine injection induced coronary artery intimal hyperplasia. (D) The nicotine injection with balloon overdilation significantly increased the intimal hyperplasia compared to simple balloon or nicotine injection groups. (E) Table shows the results of the volumetric analysis of OCT. Nicotine injection with balloon overdilation groups increased the percentage of intimal area compared to the control groups. The nicotine injection with balloon overdilation groups showed significantly increased percent intimal area compared to the other groups (26.38 \pm 3.81 in control group vs. $36.15 \pm 7.65$ in balloon overdilation group vs. $42.07 \pm 6.75$ in nicotine injection group vs. $60.86 \pm 7.20$ in nicotine with balloon overdilation group). ( ${ }^{\mathrm{P}<0.05}$ compared to control groups. \# $\mathrm{P}<0.05$ compared to balloon or nicotine injection groups). 


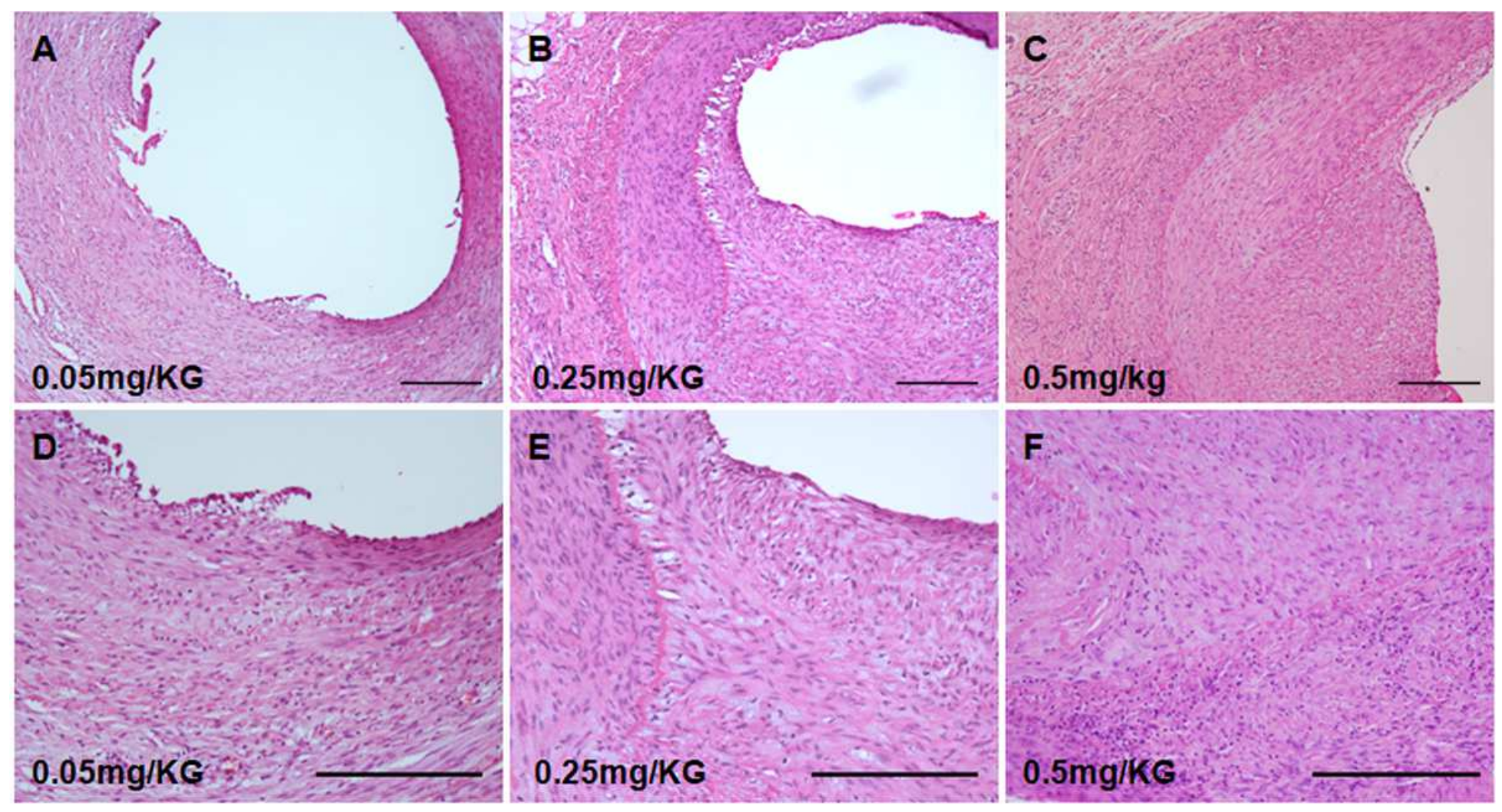

Figure 2

Hyperplasia of the coronary arteries after nicotine injection with balloon overdilation. (A, D) The dose of $0.05 \mathrm{mg} / \mathrm{kg}$ nicotine with balloon overdilation delayed the healing of the injured internal elastic lamina and induced proliferation of the tunica media. (B, E) The dose of $0.25 \mathrm{mg} / \mathrm{kg}$ nicotine injection with balloon overdilation induced clear intimal hyperplasia compared to that seen in the lower dose and simple balloon overdilation. (C, F) The dose of $0.5 \mathrm{mg} / \mathrm{kg}$ nicotine injection with balloon overdilation induced intimal hyperplasia similar to the $0.25 \mathrm{mg} / \mathrm{kg}$ nicotine injection with balloon overdilation. Bar: $100 \mu \mathrm{m}$ 


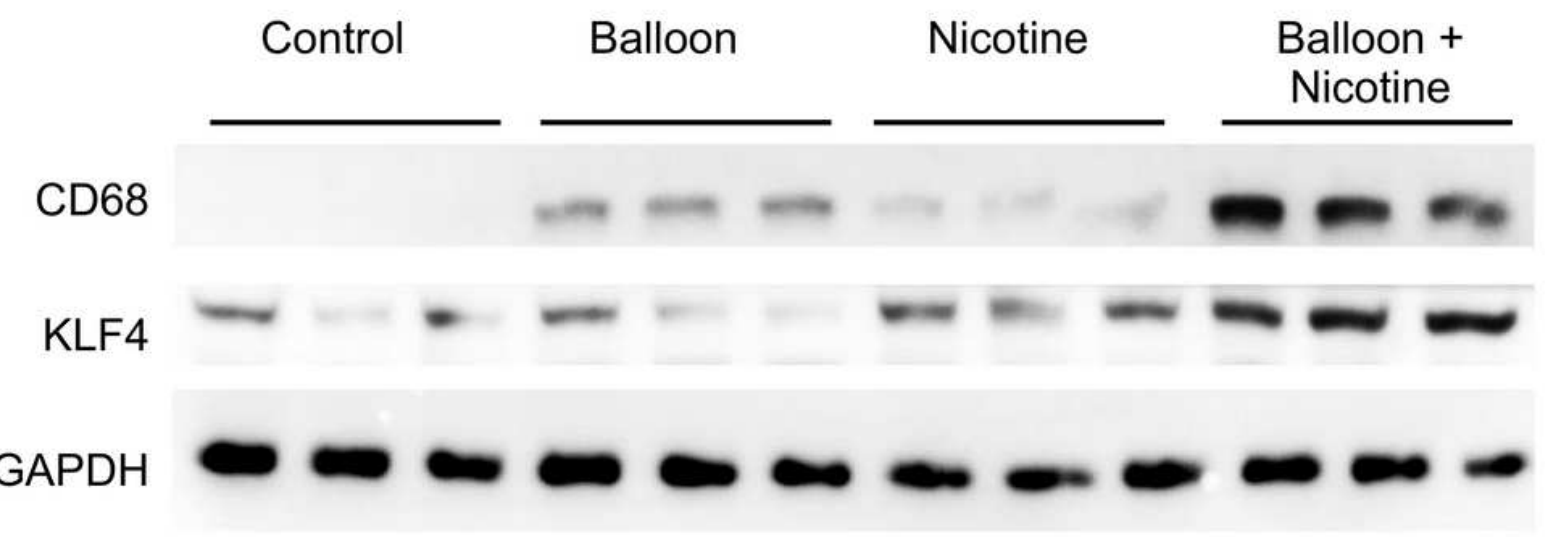

B

C
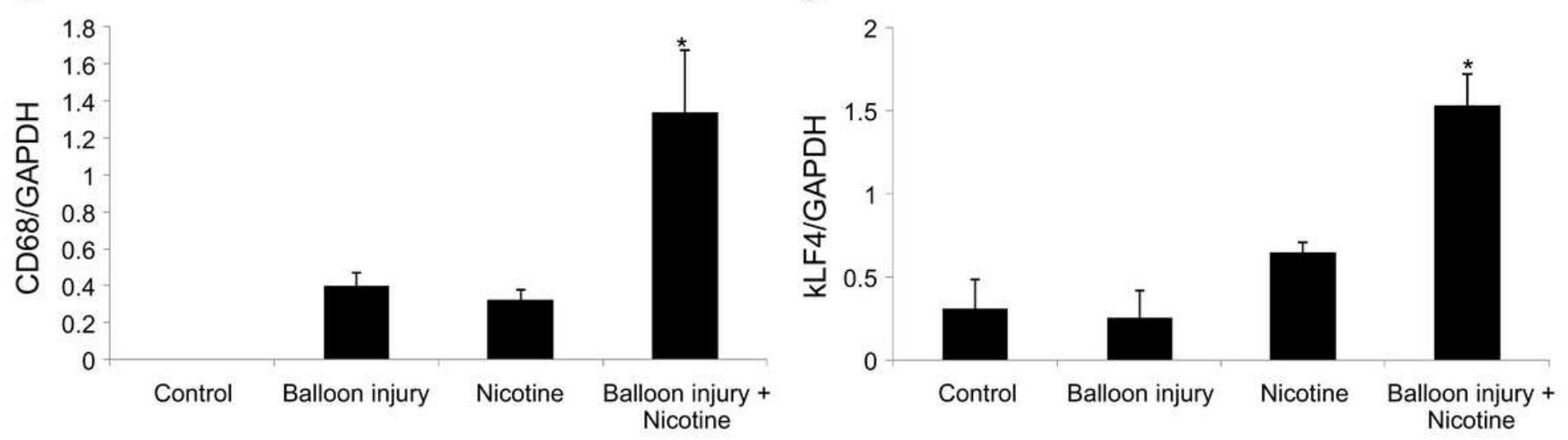

Figure 3

Increased expression of Krüppel-like factor 4 (KLF4) and cluster of differentiation 68 (CD68) in the nicotine injection with balloon overdilation groups. (A) Western blot analysis was performed to identify the changes in KLF4 and CD68 expression. (B) In the control groups, CD68 was not expressed in the coronary arteries. Balloon overdilation or nicotine injection increased CD68 expression. The nicotine injection with balloon overdilation groups had significantly increased CD68 expression compared to the other groups. (C) KLF4 expression was similar in the control and balloon overdilation groups and nicotine injection increased KLF4 expression. Nicotine injection with balloon overdilation significantly increased KLF4 expression in the coronary arteries. ${ }^{*} \mathrm{P}<0.05$. 


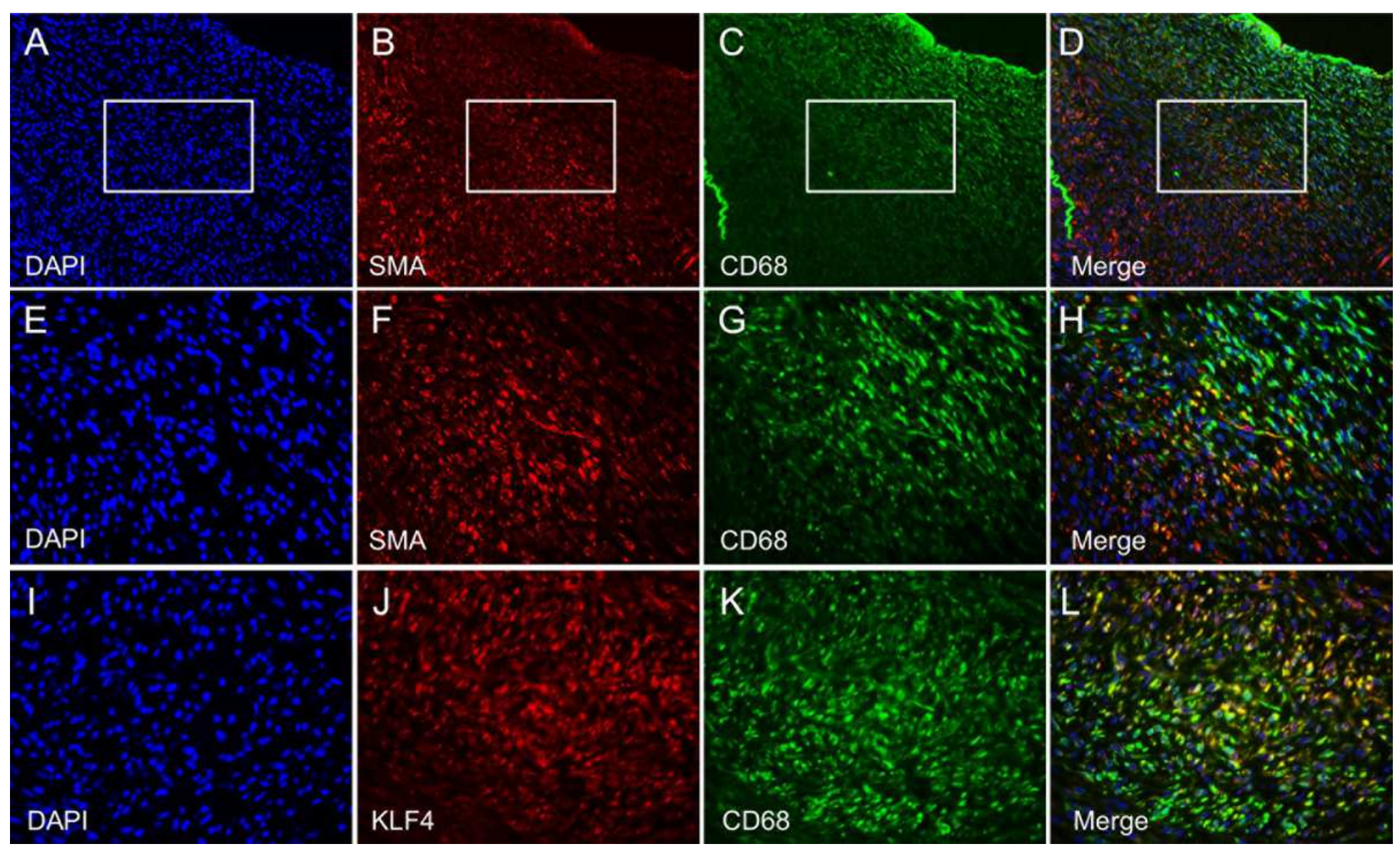

Figure 4

Alpha smooth muscle actin (SMA) positive cells and cluster of differentiation 68 (CD68) in the intimal hyperplasia lesion of nicotine injection with balloon overdilation group. (A-D) In the hyperplastic lesion of nicotine injection with balloon overdilation groups, SMA positive cells and CD68 positive cells were separately observed. (E-H) An examination at higher magnification, board zone revealed SMA and CD68 co positive cells. (I-L) CD68 positive cells co expressed KLF4.

\section{Supplementary Files}

This is a list of supplementary files associated with this preprint. Click to download.

- SupFigure1.tif

- Supplementary.pdf 